\title{
Quantification of voriconazole in plasma by liquid chromatography-tandem mass spectrometry
}

\author{
Michael Vogeser ${ }^{1, *}$, Xaver Schiel $^{2}$ and Ute \\ Spöhrer ${ }^{3}$ \\ ${ }^{1}$ Institute of Clinical Chemistry, \\ ${ }^{2}$ Department of Internal Medicine III, \\ ${ }^{3}$ Hospital Pharmacy, \\ Hospital of the University of Munich, Munich, \\ Germany
}

\begin{abstract}
A convenient liquid chromatography-tandem mass spectrometry method for the quantification of the triazole antifungal agent voriconazole in plasma samples is described. Fenbuconazole is used as an internal standard. After protein precipitation, automated solid-phase extraction is applied. Electrospray ionization in the positive mode is used and the following mass transitions are recorded: voriconazole, $350 \rightarrow 127$; and fenbuconazol, $337 \rightarrow 125$. The analytical run time is $4 \mathrm{~min}$. The response was linear from 78 to $5000 \mu \mathrm{g} / \mathrm{L}$. The total coefficient of variation $(n=16)$ was $12.6 \%$ for a low-concentration pool (143 $\mu \mathrm{g} / \mathrm{L}$ ), $4.7 \%$ for a medium-concentration pool (419 $\mu \mathrm{g} / \mathrm{L})$, and $5.0 \%$ for a high-concentration pool (4304 $\mu \mathrm{g} / \mathrm{L})$. The method is proposed for future investigations that should be performed to test the hypothesis that therapeutic drug monitoring of voriconazole is clinically useful.
\end{abstract}

Keywords: liquid chromatography-tandem mass spectrometry; solid phase extraction; therapeutic drug monitoring; voriconazole.

\section{Introduction}

Voriconazole [UK-109,496; Vfend ${ }^{\circledR}$, Pfizer (1)] is a relatively new triazole antimycotic agent that was approved in the EU in 2002 for the treatment of invasive aspergillosis and recently for first-line therapy for invasive candidiasis. Voriconazole is available for both intravenous and oral administration. The drug is extensively metabolized by the hepatic cytochrome P450 (CYP) enzymes, mainly by the isoenzymes CYP2C19, CYP2C9, and CYP3A4 (2, 3). Due to this metabolic pathway, voriconazole is prone to interaction with a very large number of drugs; compounds or conditions which induce the mentioned CYP450 isoenzymes can result in decreased voriconazole plasma

*Corresponding author: Michael Vogeser, Institute of Clinical Chemistry, Hospital of the University of Munich, Marchioninistr. 15, 81377 Munich, Germany

Phone: + 49-89-70953221, Fax: +49-89-70953240,

E-mail: michael.vogeser@med.uni-muenchen.de concentrations, whereas substrates of these enzymes can lead to increased voriconazole levels by competitive inhibition. Genetic polymorphisms of CYP450 isoenzymes (4), particularly of CYP2C19, may also have an impact on the metabolization and consequently the bioavailability of voriconazole. Voriconazole is characterized by non-linear pharmacokinetics in all species studied (5). In the case of oral application, resorption of the drug is questionable if mucositis due to antineoplastic therapy or graft-versus-host disease is suspected. Given this substantial inter- and intra-individual variability in resorption and metabolization, resulting in a wide range of trough plasma concentrations (6-8), voriconazole doubtlessly represents a candidate for individualization of dosage based on drug concentrations in blood (therapeutic drug monitoring).

Itraconazole, another triazole antimycotic drug that is now widely used for the prevention of invasive fungal infections in hemato-oncological patients, is also extensively metabolized by CYP450 enzymes and consequently a variety of drug interactions is observed; the usefulness of therapeutic monitoring of itraconazole is now widely accepted and justified by consistent data (9). In contrast, the potential benefit of therapeutic drug monitoring of voriconazole has not been studied conclusively so far. Data reported by Denning et al. (6) are consistent with a voriconazole concentration of $250 \mu \mathrm{g} / \mathrm{L}$ as a threshold for therapeutic efficacy in invasive aspergillosis. Furthermore, there is concern about hepatotoxicity associated with voriconazole concentrations above $6000 \mu \mathrm{g} / \mathrm{L}$ (10). These preliminary data doubtlessly warrant controlled prospective large-scale investigations on the usefulness of target concentration-based voriconazole treatment. For respective studies and potential future application of voriconazole measurement in a routine setting, a specific and streamlined analytical method is of essential importance. We describe here a convenient assay based on the innovative technology of liquid chromatography-tandem mass spectrometry.

\section{Materials and methods}

\section{Analytical procedure}

Chemicals and calibration materials Methanol, acetonitrile, and water were of HPLC grade (Baker, Deventer, The Netherlands). Voriconazole was donated by Pfizer Inc. (Groton, CN, USA; Figure 1A). A stock solution ( $50 \mathrm{mg} / \mathrm{L}$ ) was prepared in acetonitrile. A stock calibrator $(10,000 \mu \mathrm{g} / \mathrm{L})$ was prepared by spiking drug-free human plasma with the stock solution of voriconazole. After overnight equilibration, working calibration materials with concentrations of 5000,1000 and 100 

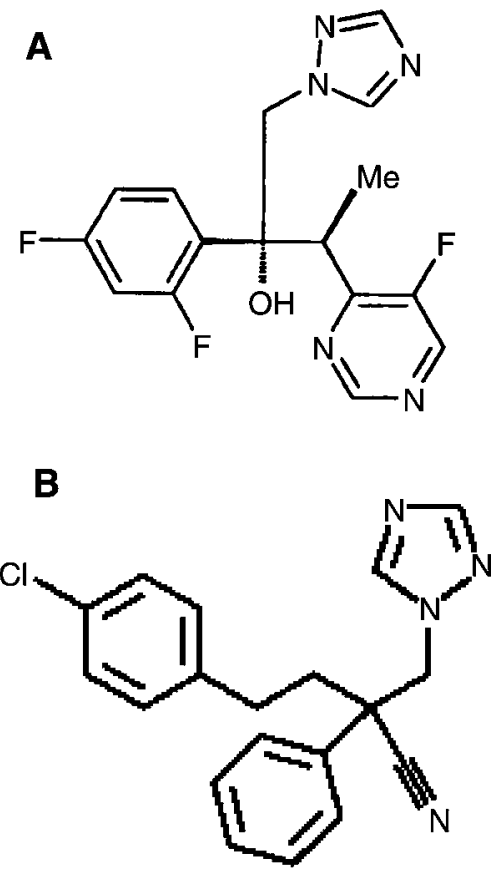

Figure 1 (A) Chemical structure of voriconazole. (B) Chemical structure of fenbuconazole, used as the internal standard compound.

$\mu \mathrm{g} / \mathrm{L}$, respectively, were prepared by dilution of the stock calibrator with drug-free plasma.

Separately, a voriconazole stock solution was diluted to prepare quality control material with a concentration of $1000 \mu \mathrm{g} / \mathrm{L}$.

For use as an internal standard, fenbuconazol (catalog no. 46096; Sigma, Deisenhofen, Germany) was used (Figure 1B). A solution of this compound with a concentration of $1 \mathrm{mg} / \mathrm{L}$ in acetonitrile was prepared (internal standard working solution, used for protein precipitation).

Instruments A Waters Alliance 2795 HPLC module (Waters, Milford, MA, USA) was used. It was coupled to a Waters Micromass Quattro LC Ultima Pt tandem mass spectrometry system with a split ratio of approximately 1:10.

Sample preparation and chromatography Aliquots of 100 $\mu L$ of patient plasma, calibrator or quality control samples, respectively, were pipeted into 2-mL polypropylene cups, then $200 \mu \mathrm{L}$ of the internal standard working solution containing $1 \mathrm{mg} / \mathrm{L}$ fenbuconazole was added. The samples were vortex mixed and centrifuged for $12 \mathrm{~min}$ at $16,000 \times \mathrm{g}$ in a standard benchtop centrifuge. Stable protein pellets were obtained and the clear supernatants were transferred to HPLC vials.

For online solid-phase extraction, a Waters Oasis HLB ${ }^{\circledR}$ column ( $25 \mu \mathrm{m}, 2.1 \times 20 \mathrm{~mm}$; Waters) was used in combination with a Rheodyne six-port high-pressure switching valve (Rheodyne, Rohnert Park, CA, USA) installed in a Waters 2795 separation module and controlled by Micromass Masslynx 4.0 mass spectrometry software.

The automated extraction procedure consisted of three steps. First, $5 \mu \mathrm{L}$ of deproteinized sample was injected and loaded onto the extraction column in valve position A (Figure 2). The mobile phase was water/methanol 95:5 (v/v) delivered at a flow rate of $4.0 \mathrm{~mL} / \mathrm{min}$; potentially interfering compounds were washed into the waste. In parallel, the analytical column (LiCrospher ${ }^{\circledR} 100$ RP-18 end-capped, $125 \times 4 \mathrm{~mm}, 5 \mu \mathrm{m}$; Merck, Darmstadt, Germany) was equili- brated with $90 \%$ methanol $/ 10 \%$ formic acid $0.1 \%(\mathrm{v} / \mathrm{v})$ delivered at a flow rate of $0.85 \mathrm{~mL} / \mathrm{min}$. After 1 min the switching valve was changed to position $B$. The extraction column was then eluted in backflush mode onto the analytical column. After $2 \mathrm{~min}$, the valve was switched back to position A. During analytical chromatography into the mass spectrometer in position $A$, the extraction column was washed with acetonitrile/methanol 50:50 (v/v) at a flow rate of $4.0 \mathrm{~mL} / \mathrm{min}$ for $1 \mathrm{~min}$ and subsequently re-equilibrated with water/methanol 95:5 (v/v). The extraction and analytical columns were maintained at $30^{\circ} \mathrm{C}$ in a column oven. The retention times of voriconazole and fenbuconazole (internal standard) were approximately $2.0 \mathrm{~min}$ after injection into the extraction column. The total analytical run time was $4 \mathrm{~min}$.

Mass spectrometric conditions Electrospray atmosphericpressure ionization in the positive mode was used. The source parameters were set to obtain the protonated quasimolecular ions of voriconazole and fenbuconazole (350 and $337 \mathrm{~m} / \mathrm{z}$, respectively). The following settings gave optimum ion yield: capillary voltage, $3.9 \mathrm{kV}$; cone voltage, $57 \mathrm{~V}$; source temperature, $90^{\circ} \mathrm{C}$; desolvation temperature, $280^{\circ} \mathrm{C}$; nitrogen flow, approximately $550 \mathrm{~L} / \mathrm{h}$; and cone gas flow, approximately $75 \mathrm{~L} / \mathrm{h}$. The collision energy was $25 \mathrm{~V}$ with argon as the collision gas. Under these conditions, several product ions were generated from the analyte and the internal standard compound. For quantification purposes, the following transitions were recorded: voriconazole, $350 \rightarrow 127$; and fenbuconazole, $337 \rightarrow 125$. The dwell time for all multiple reaction monitoring (MRM) traces was $0.3 \mathrm{~s}$; the interchannel delay and interscan delay were $0.15 \mathrm{~s}$.

A representative chromatogram obtained from a pooled plasma sample is given in Figure 3.

\section{Assay validation}

To study the recovery of the two-step extraction procedure (protein precipitation followed by online solid-phase extraction), pure solutions of voriconazole and the internal standard fenbuconazole, respectively, dissolved in acetonitrile (each $1000 \mu \mathrm{g} / \mathrm{L}$ ) were analyzed directly by HPLC and tandem mass spectrometry in triplicate; the peak areas of the two respective MRM chromatograms were recorded. For comparison, drug-free plasma was spiked with the two compounds to the same concentrations. These samples were submitted to the entire method, including protein precipitation and online solid-phase extraction, in triplicate. The peak areas found for these spiked samples were compared with those found for the pure solutions directly analyzed without extraction. From the respective mean areas from both experiments, the recovery rate was calculated.

To test the method for potential ion suppression effects, five different plasma samples from patients not treated with voriconazole were spiked to a concentration of $1000 \mu \mathrm{g} / \mathrm{L}$ voriconazole and submitted to the entire method. For comparison, a pure solution of voriconazole at a concentration of $1000 \mu \mathrm{g} / \mathrm{L}$ in acetonitrile/water $(50: 50 \mathrm{v} / \mathrm{v})$ was analyzed in the same way five times. The respective MRM peak areas for voriconazole and fenbuconazole used as the internal standard were compared.

To study the linearity of the method, a drug-free plasma sample was spiked to a concentration of $5000 \mu \mathrm{g} / \mathrm{L}$ with voriconazole; this sample was serially diluted 1:1 with drug-free plasma in six steps. The concentration of voriconazole was measured in all samples. The measured and expected concentrations were compared.

To study the imprecision of the method, plasma pools were prepared from residual clinical samples from vorico- 
A

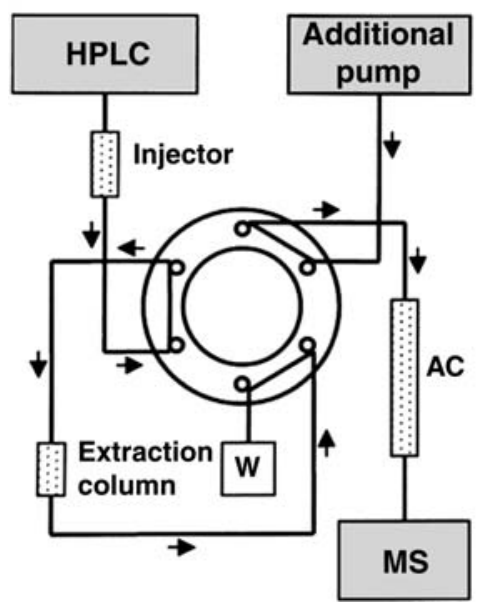

B

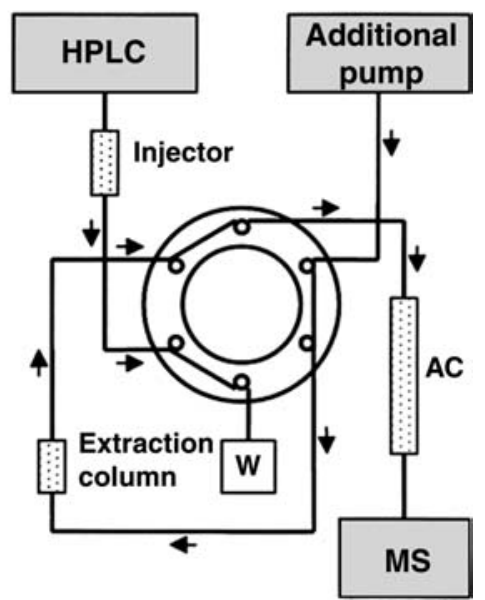

Figure 2 Column switching scheme used for online solid-phase extraction of voriconazole (W, waste; AC, analytical column).

nazole-treated patients at high, medium, and low concentration ranges $(143,419$ and $4304 \mu \mathrm{g} / \mathrm{L})$. These pools were equilibrated overnight, aliquoted, and stored at $-80^{\circ} \mathrm{C}$. In four independent analytical series, each pool was analyzed four times to calculate the respective intra-assay coefficients of variation. A total coefficient of variation was calculated for each pool from all 16 results obtained in the four analytical series.

\section{Results}

A recovery rate of $96 \%$ for the entire extraction procedure, including manual deproteinization and automated online solid-phase extraction, was found. No ion suppression was noted by comparison of the MRM peak areas for voriconazole and the internal standard, respectively, found in pure solution samples and human plasma samples spiked to the same concentration; there was no significant difference in the respective peak areas.

The results of the linearity investigation are given in Table 1. Linear regression analysis of these data revealed a coefficient of correlation of $r^{2}=0.999$ between expected and observed voriconazole concentrations, confirming linearity of the assay for plasma concentrations between 78 and $5000 \mu \mathrm{g} / \mathrm{L}$.

In the imprecision study, the following mean concentrations were found, with the respective total coefficient of variation $(n=16)$ : low pool, $143 \mu \mathrm{g} / \mathrm{L}, 12.6 \%$ (intra-assay CV range 1.0-9.7\%); medium pool, $419 \mu \mathrm{g} / \mathrm{L}, 4.7 \%$ (intra-assay CV range 0.7-3.8\%); high

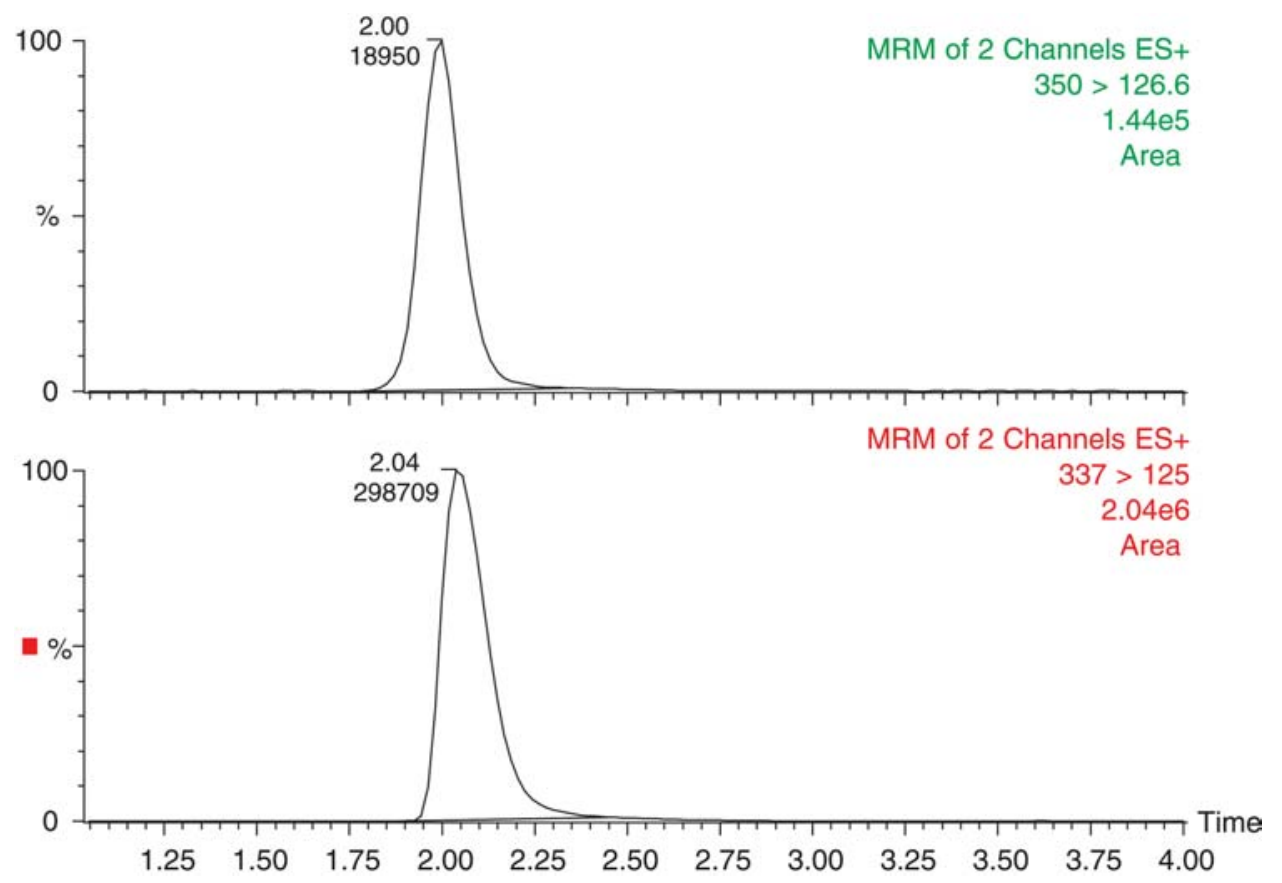

Figure 3 Representative MRM chromatogram of voriconazole $(350>126.6)$ and fenbuconazole $(337>125)$ used as the internal standard (concentration of voriconazole, $143 \mu \mathrm{g} / \mathrm{L}$; the $\mathrm{Y}$-axis represents the relative intensity of the signal with respect to the base peak of the chromatogram). 
Table 1 Results of the linearity investigation (serial dilution of a spiked voriconazole sample with drug-free plasma).

\begin{tabular}{llc}
\hline Concentration, $\mu \mathrm{g} / \mathrm{L}$ & & Accuracy, \% \\
\cline { 1 - 2 } Expected & Measured & \\
\hline 5000 & 4865 & 97 \\
2500 & 2561 & 102 \\
1250 & 1303 & 104 \\
612 & 623 & 102 \\
306 & 287 & 94 \\
153 & 163 & 107 \\
75 & 78 & 104 \\
0 & 0 & \\
\hline
\end{tabular}

pool, $4304 \mu \mathrm{g} / \mathrm{L}, \quad 5.0 \%$ (intra-assay $\mathrm{CV}$ range 1.4-3.2\%).

For the quality control sample with a spiked concentration of $1000 \mu \mathrm{g} / \mathrm{L}$, a mean accuracy of $98.3 \%$ was found in the analytical series performed during method validation.

\section{Discussion}

In this article a convenient liquid chromatographytandem mass spectrometry method for the quantification of plasma voriconazole concentrations is described. The method uses automated online solidphase extraction with column switching and a polymeric extraction material; the related antimycotic compound fenbuconazole is used as the internal standard. Assay validation demonstrated good analytical performance of the method. Based on the imprecision study, a concentration of $150 \mu \mathrm{g} / \mathrm{L}$ is recommended as the lower limit of quantification of the method.

HPLC methods for the quantification of voriconazole using UV detection have been described previously (11-13). However, UV detection is a nonspecific technique. In particular, in patients with organ failure and co-administration of multiple drugs - as applies to most patients receiving voriconazole - the risk of co-elution of unspecified endogenous or exogenous compounds together with voriconazole or a respective internal standard substance in HPLC methods is substantial, and false results could be obtained by applying UV detection. Since mass spectrometric detection for HPLC has been developed as a routine analytical technique in the clinical laboratory $(14,15)$, such very specific methods should be applied whenever possible.

Very recently a method for serum voriconazole measurement based on liquid chromatography-tandem mass spectrometry has been published by Keevil et al. (16). The assay described here, however, differs essentially from this previous method, namely in the mode of analyte extraction and in the internal standard used.

In the method described by Keevil et al. (16) a simple variation of online solid-phase extraction is applied that does not use a separate extraction column. In this method the analyte - bound to the analytical column after injection of precipitated sample - is washed with only $300 \mu \mathrm{L}$ of an aqueous solution and subsequently eluted with methanol; the column eluate is then transferred to the mass spectrometer without solvent diversion. Using this "pre-chromatography" without column switching, the target analyte is separated from residual matrix components, thus reducing ion suppression effects. However, throughout the whole analytical cycle, all matrix components that remain after protein precipitation of the sample enter the ion source and can cause contamination. Therefore, the robustness of this method during extended series in a routine setting is questionable. In the method described here, the sample - after protein precipitation - is loaded onto a co-polymeric extraction material packed in a separate extraction column. This column is subsequently washed within $1 \mathrm{~min}$ with $5 \%$ methanol in water at a very high flow rate, resulting in a washing volume of $4 \mathrm{~mL}$. After column switching and backflush elution of the analyte onto the separate analytical column, the extraction column is washed with a high volume of an organic solvent mix. Using this procedure, a very clean extract is submitted to analytical chromatography and finally transferred in split mode to the electrospray ion source. The co-polymeric extraction material used (Waters Oasis HLB) allows very high flow rates, resulting in very efficient washing within a short time. We have excellent experience with this extraction protocol, which has been applied for several years in the analysis of immunosuppressants in whole blood and itraconazole in plasma in our laboratory $(17,18)$. The lifetime of the extraction and analytical columns is several thousands of analyses. Minimal deposits are found on the ion source components after several thousands of analyses and the lifetime of the electrospray capillary is very satisfactory. Online solidphase extraction after protein precipitation using column switching and the Waters Oasis HLB material is an extremely convenient, fast, and proven technique with generic applicability for lipophilic drugs.

The application of an appropriate internal standard compound is of essential importance for reliable and precise LC-MS methods. In conventional HPLC methods, internal standardization is mainly necessary to compensate for between-sample variations in the efficacy of the extraction and imprecision of the HPLC injector. In contrast, in liquid chromatography methods using atmospheric-pressure ionization, internal standardization must moreover compensate for variations in the ionization efficacy from sample to sample and within a series. The ion yield of atmospheric-pressure ionization techniques typically undulates within seconds or minutes and often shows trends within hours. Variations in the ion yield can differ substantially between different compounds. It is essential and critical for the precision of an LC-MS method that the short-term variation of the ionization yield of a target analyte and the respective internal standard due to source variables such as contamination is affected in a very similar manner. This is ideally given by stable isotope-labeled internal standards; however, these are only available for a few 
drugs. In all other cases it is essential to find a compound with a molecular structure that is very closely related to that of the target analyte. Keevil et al. (16) used ketoconazole as the internal standard. This compound is also a triazole antimycotic drug; however, its molecular structure is quite different from that of voriconazole, as evident from the molecular mass (voriconazole, 349; ketoconazole, 530). Instead of ketoconazole, we use the agrochemical fenbuconazole for internal standardization; its molecular structure is very similar to that of voriconazole (Figure 1). Assay validation data reported by Keevil et al. are in close agreement with those found in our investigation. However, it must be assumed from the abovementioned considerations that the use of the very closely related compound fenbuconazole instead of the far less similar compound ketoconazole results in superior long-term stability and robustness of an LCMS method for the quantification of voriconazole in biological samples in a routine setting.

Principal considerations and preliminary epidemiological data (6) point to the need for therapeutic drug monitoring of voriconazole. Doubtlessly, further controlled large-scale clinical studies are needed to clarify the potential benefit of individualization of voriconazole treatment based on blood drug concentrations. For further investigations, we propose the convenient and rugged LC-MS method for voriconazole measurement described in this report.

\section{References}

1. Johnson LB, Kauffman CA. Voriconazole: a new triazole antifungal agent. Clin Infect Dis 2003;36:630-7.

2. Hyland R, Jones BC, Smith DA. Identification of the cytochrome P450 enzymes involved in the N-oxidation of voriconazole. Drug Metab Dispos 2003;31:540-7.

3. Voriconazole FDA briefing document, 2001 (http:// www.fda.gov/ohrms/dockets/ac/01/briefing/3792b2_01_Pfi zer.pdf).

4. Ozawa S, Soyama A, Saeki M, Fukushima-Uesaka H, Itoda $\mathrm{M}$, Koyano S, et al. Ethnic differences in genetic polymorphisms of CYP2D6, CYP2C19, CYP3As and MDR1/ ABCB1. Drug Metab Pharmacokinet 2004;19:83-95.

5. Roffey SJ, Cole S, Comby P, Gibson D, Jezequel SG, Nedderman $\mathrm{AN}$, et al. The disposition of voriconazole in mouse, rat, rabbit, guinea pig, dog, and human. Drug Metab Dispos 2003;31:731-41.
6. Denning DW, Ribaud P, Milpied N, Caillot D, Herbrecht $R$, Thiel $E$, et al. Efficacy and safety of voriconazole in the treatment of acute invasive aspergillosis. Clin Infect Dis 2002;34:563-71.

7. Boyd AE, Modi S, Howard SJ, Moore CB, Keevil BG, Denning DW. Adverse reactions to voriconazole. Clin Infect Dis 2004;39:1241-4.

8. Trifilio S, Ortiz R, Pennick G, Verma A, Pi J, Stosor V, et al. Voriconazole therapeutic drug monitoring in allogeneic hematopoietic stem cell transplant recipients. Bone Marrow Transplant 2005;35:509-13.

9. Glasmacher A, Hahn C, Leutner C, Molitor E, Wardelmann E, Losem C, et al. Breakthrough invasive fungal infections in neutropenic patients after prophylaxis with itraconazole. Mycoses 1999;42:443-51.

10. Potoski BA, Brown J. The safety of voriconazole. Clin Infect Dis 2002;35:1273-5.

11. Stopher DA, Gage R. Determination of a new antifungal agent, voriconazole, by multidimensional high-performance liquid chromatography with direct plasma injection onto a size-exclusion column. J Chromatogr B Biomed Sci Appl 1997;691:441-8.

12. Gage R, Stopher DA. A rapid HPLC assay for voriconazole in human plasma. J Pharm Biomed Anal 1998; 17:1449-53.

13. Pennick GJ, Clark M, Sutton DA, Rinaldi MG. Development and validation of a high-performance liquid chromatography assay for voriconazole. Antimicrob Agents Chemother 2003;47:2348-50.

14. Dooley KC. Tandem mass spectrometry in the clinical chemistry laboratory. Clin Biochem 2003;36:471-81.

15. Vogeser M. Liquid-chromatography tandem mass-spectrometry - application in the clinical laboratory. Clin Chem Lab Med 2003;41:117-26.

16. Keevil BG, Newman S, Lockhart S, Howard SJ, Moore CB, Denning DW. Validation of an assay for voriconazole in serum samples using liquid chromatography-tandem mass spectrometry. Ther Drug Monit 2004;26:650-7.

17. Vogeser M, Fleischer C, Meiser B, Groetzner J, Spöhrer $U$, Seidel D. Quantification of sirolimus by liquid chromatography-tandem mass spectrometry using on-line solid-phase extraction. Clin Chem Lab Med 2002;40: 40-5.

18. Vogeser M, Spöhrer U, Schiel X. Determination of itraconazole and hydroxyitraconazole in plasma by use of liquid chromatography-tandem mass spectrometry with on-line solid-phase extraction. Clin Chem Lab Med 2003;41:915-20.

Received March 15, 2005, accepted April 29, 2005 